\title{
Recension des Congrès de la Société Française Shakespeare
}

Jean-Pierre Villquin

\section{OpenEdition}

Journals

Édition électronique

URL : http://journals.openedition.org/shakespeare/1582

DOI : 10.4000/shakespeare.1582

ISSN : 2271-6424

Éditeur

Société Française Shakespeare

Édition imprimée

Date de publication : 1 novembre 1997

Pagination : 195-208

Référence électronique

Jean-Pierre Villquin, «Recension des Congrès de la Société Française Shakespeare », Actes des congrès de la Société française Shakespeare [En ligne], 15 | 1997, mis en ligne le 01 janvier 2007, consulté le 06 mai 2019. URL : http://journals.openedition.org/shakespeare/1582 ; DOI : 10.4000/ shakespeare.1582 


\section{A Q U E S T IOON D U M A L E T L E M A L E N QUESTION DA NS HAMLET}

«No man means evil but the devil» $(M . W .$, V.2.13)

«Personne ne songe à mal si ce n'est le démon», cette réplique de George Page dans The Merry Wives que je mets en exergue semble, sur le ton de la plaisanterie, innocenter l'homme du péché originel, donc du mal. Cet acquittement n'implique pas que le mal puisse exister dans l'absolu, d'ailleurs il ne se définit la plupart du temps que par rapport au bien, comme l'ombre s'oppose à la lumière, ce qui est une façon d'éluder le problème. Et pourtant le mal est venu aux hommes. Dans une pièce comme Hamlet, ${ }^{1}$ le mal est partout. Il est à la fois une question, la question, et une mise en question. «S le mal ne suscitait la question : pourquoi le mal ? il ne serait pas le mal... S la réponse à la question : pourquoi ? allait de soi dans une évidence naturelle, le mal disparaîtrait en même temps que serait effacée l'angoisse de la question» ${ }^{2}$. Or que fait Hamlet, sinon s'interroger sans cesse et nous prendre à témoin de ses angoisses? Hamlet découvre l'univers du mal par personne interposée, le mot personne n'est peut-être pas exact puisqu'il s'agit d'un fantôme. Hamlet doit accomplir une mission de vengeance, mission paradoxale car elle met en jeu une forme de justice qui rend le mal pour le mal, et l'inscrit dans un cycle perpétuel. Hamlet est confronté à la réalité du mal dans le monde et chez ceux qui l'entourent. Son combat est la nécessaire reconnaissance et même la nécessaire acceptation de ce mal dans la mesure où lui-même le partage puisqu'il est homme et «porte en lui la forme entière de 
l'humaine condition». Cette quête négative pour retarder et pour rejeter le mal aboutit à une impasse apparente : la mort d'Hamlet, mais aussi à une amère victoire remportée par un équivoque Fortinbras. Les questions posées resteront - heureusement pour les exégètes présents et à venir - sans réponse définitive, mais je vous invite cependant à explorer quelques pistes qui nous permettront d'abord de constater la présence du mal dans la tragédie, ensuite d'appréhender le problème de la conscience du mal, et enfin de nous interroger sur la dramaturgie et l'esthétique du mal.

\section{LA PRÉSENCE DU MAL}

\section{A. Le mal commis}

Le meurtre rapporté, imaginé et surtout représenté sur scène, fait évidemment partie des éléments les plus spectaculaires de la tragédie. L'assassinat prémédité du vieil Hamlet s'apparente au meurtre fondateur, celui d'Abel par son frère Caïn. Aucun détail n'est épargné, ni les mobiles du crime de Claudius : l'ambition, la couronne de Danemark et sa passion pour la reine, ni les moyens utilisés : le poison versé dans l'oreille (les oreilles), ni le lieu ni le temps. L'assassinat du jeune Hamlet est lui aussi prémédité par ce même Claudius qui envoie son neveu se faire exécuter en Angleterre, qui prépare un duel truqué, qui prévoit des moyens de substitution et qui s'assure de la complicité complaisante de Laertes. Ce meurtre par intérim n'est pas l'apanage du scélérat conventionnel car Hamlet, avec, il est vrai, le bénéfice des circonstances atténuantes que constitue la légitime défense, programme lui aussi dans les moindres détails la mort de Rosencrantz et de Guildenstern, sans même leur accorder le temps de se confesser (V.2.46-8).

Si la préméditation aggrave ces représentations du mal, le hasard, le destin ou la fatalité apparaissent aussi comme des agents actifs du mal. C'est par exemple le coup d'épée à travers la tapisserie qui laisse sans vie Polonius (III.4.29-30 et didascalie 25-6). C'est le hasard qui cause la mort de Gertrude buvant le poison qui ne lui était pas destiné. C'est enfin le hasard qui fait que Hamlet et Laertes échangent leurs épées.

L'acharnement d'Hamlet forçant Claudius déjà mortellement blessé à boire le poison n'est pas loin de cette forme extrême du mal qu'est la perversité. Les mots autant que les actes peuvent être aussi dangereux que des poignards. La cruauté mentale peut être si violente qu'elle s'apparente à la cruauté physique au point que Gertrude craint pour sa vie (III.4.22). Avec sa mère comme avec Ophélie, Hamlet cherche à blesser au plus profond. S'il semble avoir des griefs, fondés à ses yeux, pour persécuter sa mère, il n'y a guère de justification pour sa cruauté envers Ophélie, sinon sa condition de femme, ce «vaisseau d'infirmité» descendante de la première pécheresse. L'esprit de vengeance, c'est-à-dire rendre le mal pour le mal prend des formes diverses. Il peut être spontané et violent comme chez Laertes qui renonce à la 
religion et à son sens du bien et du mal pour se donner au démon le plus noir (IV.5.131-3), il peut être calculé comme chez Claudius invoquant la raison d'État, il peut aussi être délégué comme la vengeance du vieil Hamlet. Dans tous les cas la violence est présente, en paroles et en actes (V.1.236-9). Les lieux les plus sacrés ne peuvent même pas servir de sanctuaire car nul sanctuaire ne protège le meurtre, et la vengeance ne doit pas connaître de limites (IV.7.102-3).

A tous ces meurtres individuels, on peut ajouter les meurtres collectifs et licites que sont la guerre ensanglantant la Pologne, les luttes ancestrales comme celles du vieil Hamlet et du vieux Fortinbras, ou le contentieux entre l'Angleterre et le Danemark.

\section{B. Le mal subi}

Le mal n'est pas seulement incarné par les auteurs du mal, il l'est aussi par les victimes. Le rôle de bourreau et celui de victime se confondent parfois, mais le principe de justice immanente qui prévaut dans le genre tragique s'applique évidemment dans Hamlet. Il y a d'abord les victimes innocentes, et puisque je viens d'évoquer la guerre, les soldats en sont les premières : vingt mille hommes peuvent mourir pour un lopin de terre et la gloire dérisoire de leur chef (appendice, après IV.4.8). Il y a aussi les enfants, héritiers de querelles auxquelles ils sont étrangers. Le mal n'est pas fait que de crimes inexpiables, de malheurs insurmontables, il est aussi fait de tous les maux quotidiens qui rendent la vie insupportable. Pour Ophélie c'est la douleur de voir son amour bafoué, ses espérances anéanties par Hamlet, par son père et par son frère. C'est aussi le spectacle du malheur des autres, la folie d'Ophélie, ou celle d'Hamlet. Porter les erreurs, les fautes et les péchés d'autrui est pour ce dernier un trop lourd fardeau. Gertrude a trahi ceux qui lui étaient les plus proches et a transgressé les codes les plus sacrés. Elle est déchirée entre ses sentiments de mère et la vision imposée d'une faute qu'elle ne perçoit qu'à moitié. Claudius lui-même n'est pas inaccessible au remords et souffre au souvenir de son crime. Mais c'est évidemment Hamlet dont le mal de vivre est le plus vif. Dans tous ces cas c'est le mal intérieur qui ronge l'âme des vivants comme les vers rongent le corps des morts.

Dans Hamlet il y a peu de souffrance physique, il n'y a pas de tortures comme l'exécution scandaleuse d'un roi, pas de honteuse fistule, pas de tempêtes meurtrières, mais un mal latent, insidieux, permanent qui se glisse partout.

\section{Hamlet ou le royaume du mal}

Si au théâtre, un concept abstrait comme le mal est principalement représenté dans les personnages qui l'incarnent ou qui en sont les victimes, Hamlet apparaît comme un palimpseste sur lequel aurait été écrite l'histoire du 
mal. Le Danemark est son royaume. Le chaos règne et la révolte menace (I.1.69). A l'extérieur on pense que l'État est disloqué, fragile, prêt à s'effondrer (I.2.20). L'atmosphère est corrompue et tout peut arriver (I.2.257 et 259). Le château d'Elseneur est une prison (II.2.242-3). Comme un vers dans le fruit, le mal est à l'intérieur de chacun, ronge et contamine l'âme et le corps (III.4.143-6). Le mal, la maladie et le péché sont toujours étroitement associés. Il ne faut donc pas être surpris des images récurrentes de la mort, des cimetières, et du processus de décomposition. La mort, forme du mal absolu, inéluctable, fatale, devient une banalité (I.2.72).

L'univers d'Hamlet est celui du paradis perdu où règne le désordre, où poussent les herbes folles :

[this world] 'Tis an unweeded garden

That grows to seed; things rank and gross in nature Possess it merely. That it should come to this !

S'ils ne sont pas présentés comme un spectacle allégorique, les péchés capitaux et leurs variantes sont incarnés dans les personnages. Claudius est le scélérat, le démon, le mal (II.2.568-9), il passe ses nuits à faire carousse, à vider des hanaps de vin du Rhin au cours de fêtes orgiaques (I.4.8-10 et III.2.284-6). Il se livre à la luxure et commet l'inceste, souillant ainsi la dignité de roi (I.5.74-9). Le vieil Hamlet n'est pas innocent, s'il est condamné à l'enfer, c'est qu'il est mort sans être pardonné de ses péchés et sans avoir sacrifié aux rites chrétiens :

Cut off even in the blossom of my sin

Unhouseled, dis-appointed, unaneled,

No reckoning made, but sent to my account

With all my imperfections on my head.

(I.5.76-9)

Hamlet lui-même semble prendre un malin plaisir à son statut de pécheur à l'honnêteté douteuse, orgueilleux, vindicatif et ambitieux, et si les femmes ne sont bonnes qu'à engendrer des pécheurs, lui n'est qu'un homme, un pauvre pécheur, et les hommes ne sont-ils pas tous des scélérats ?: «Get thee to a nunnery. Why, wouldst thou be a breeder of sinners ? I am myself indifferent honest... I am very proud, revengeful, ambitious... What should such fellows as I do crawling between heaven and earth ? We are arrant knaves all» (III.1.122-130). Les deux seules femmes qui apparaissent dans Hamlet sont d'abord les héritières d'Ève, cause du péché originel, et elles sont toujours 
marquées de tous les stigmates des faiblesses de leur sexe, coquettes, pernicieuses et fragiles (I.2.146; III.1.145-7 et I.5.104-7).

Les images évoquent le démon et les flammes de l'enfer. Le fantôme brosse un tableau saisissant de ses tourments dans l'attente du jugement dernier (II.2.237), condamné à errer le jour dans sa prison de flammes tant que ses fautes ne seront pas lavées (I.5.2-4 et I.5.9-13). Le palais d'Elseneur, les champs de bataille de Pologne, la futilité de la vie parisienne, bref, le monde des hommes n'est que le reflet de l'enfer, et les cimetières qui bâillent nous rappellent l'univers souterrain qui exhale les miasmes sulfureux et révèle ce qui reste des morts (III.2.371-3).

Le mal et la notion de mal sont donc bien présents dans Hamlet. Cette notion se définit plus par rapport à elle-même que par rapport au bien qui est pratiquement absent. Il n'y a pas véritablement de distinction entre le mal physique, principalement la mort, le mal moral et le mal métaphysique, ce sont là des distinctions souvent artificielles, car le mal coule d'une même source, il a une histoire, il s'inscrit dans ses mythes. Avec ses références à Caïn et au péché originel, Shakespeare nous renvoie à la Genèse, mais semble faire un choix délibéré entre les deux récits de la création. Dans le premier récit, Dieu fait l'homme à son image et lui donne pour nourriture toutes les herbes portant semence et tous les arbres qui ont des fruits, sans restriction. Dans cette version le mal n'existe pas, ou tout au moins n'est pas mentionné, et par nature l'homme ressemble à Dieu. Cette vision ne se retrouve pas dans Hamlet, et le mal, dans la tragédie, doit davantage à la deuxième version de la création du monde qu'on peut résumer ainsi : Yahvé créa l'homme à partir de la glaise, le plaça dans un jardin en Éden où poussaient l'arbre de vie et l'arbre de la connaissance du bien et du mal. Sous peine de mort, Dieu interdit à l'homme de manger les fruits de cet arbre ${ }^{3}$. Séduite par les paroles du serpent, la femme mangea le fruit défendu et ainsi l'homme devint semblable à Dieu pour distinguer le bien du mal. Le pécheur s'est ainsi arrogé la fonction de juge, ce qui était l'apanage de Dieu. (Gen.2.7-17 et 3.4-22). Dieu punit l'homme pour sa désobéissance et le mal se répandit sur la terre. Le meurtre fondateur (Gen.4.8), première manifestation du mal, fut commis quand Caïn tua son frère Abel (V.1.73-4) et depuis, le mal habite l'homme et la femme qui en est la principale vectrice et la propagatrice.

Une seule tare, un défaut de naissance, la moindre imperfection peut discréditer un homme : "Une goutte de mal noircit souvent la plus noble substance». Mais s'il n'est pas responsable de sa naissance, peut-il être coupable? (appendice A (i), 7-16). Si l'homme est prédestiné, marqué du sceau du péché, s'il est à la merci des étoiles contraires ou des dieux capricieux, que peut-il faire ? Il est troublé, angoissé, ébranlé, paralysé par le mystère du mal qui le dépasse : «With thoughts beyond the reaches of our souls ?» (I.4.35) 


\section{LA CONSCIENCE DU MAL}

\section{A. La loi de Dieu, la loi des hommes}

L'arbre de la connaissance a donc permis aux hommes de distinguer entre le bien et le mal, de distinguer l'un et l'autre, ou plutôt l'un par l'autre. Shakespeare ajoute la notion de relativité et d'équivoque, car chacun juge à l'aune de son expérience. Elseneur peut être un palais pour Rosencrantz et Guildenstern, mais c'est une prison pour Hamlet, car rien n'est bon ni mauvais en soi, mais dépend de la façon de voir de chacun : «for there is nothing either good or bad, but thinking makes it so» (II.2.248-9). La conscience du bien et du mal peut être une froide réflexion, comme c'est le cas pour Claudius croyant être seul avec lui-même. Elle peut conduire à une plus grande confusion par le feu croisé d'interrogations comme chez Hamlet, enfin elle peut être imposée brutalement comme elle l'est à Gertrude par son fils qui lui tend un miroir afin de lui montrer le plus secret d'elle-même : «a glass / Where you may see the inmost part of you» (III.4.20-1).

Le péché, la faute, la transgression des lois de Dieu sont donc mis en évidence, mais ils sont relatifs. Chacun fait sa propre lecture. Hamlet accuse sa mère qui lui demande avec une innocence feinte ou réelle quel acte répréhensible elle a pu commettre : «Ay me, what act, / That roars so loud and thunders in the index ?» (III.4.53-4), et son fils lui rappelle que la faute originelle est de succomber à la tentation. Celle-ci peut prendre toutes les formes, et sous le masque de la religion et des pieuses pratiques, c'est le diable qui cache nos mines sucrées comme le dit Polonius (III.1.47). Horatio imagine un instant que le fantôme peut être un démon qui risque d'entraîner Hamlet (I.4.48 \& 51). Gertrude s'est laissée prendre aux séductions et au jeu du serpent qu'est Claudius : «What devil was't / That thus hath cozened you at hoodman's blind ?» (III.4.70-3). La naïveté d'Ophélie est aussi une forme d'erreur, de faute, même si Hamlet semble avoir respecté les formes et les rites, elle s'est laissée prendre au piège de la séduction : «And hath given countenance to his speech, my lord, / With almost all the holy vows of heaven» (I.3.113-4). Le «presque» entache la sincérité de Hamlet et laisse penser que le démon n'est pas absent chez celui qui aime à se faire passer pour un redresseur de torts.

Les péchés capitaux sont encore plus évidents : le meurtre d'un frère ou un mariage incestueux aggravé par une hâte indécente. Rien de bon ne peut venir d'actes mauvais (I.2.156-8). Cependant la vengeance et la justice des hommes ont leurs limites et le fantôme rappelle à Hamlet que s'il lui confie la mission de le venger de Claudius, il doit laisser au ciel celle de punir la pécheresse qu'est sa mère : «leave her to heaven, / And to those thorns that in her bosom lodge / To prick and sting her» (I.5.82-88).

La conscience aiguë du mal conduit les plus pervers à s'interroger sur la gravité de leur faute et à envisager une éventuelle absolution. Claudius se 
conduit en casuiste retors quand il se fait, si on peut dire, l'avocat du diable pour montrer que si Dieu l'a soumis à la tentation et n'a pas su le retenir de pécher, sa responsabilité d'homme n'est pas complètement engagée, et dans ces conditions, c'est à Dieu de lui pardonner. C'est le même raisonnement pervers qui conduit Hamlet à s'exonérer de la mort de Polonius en mettant ce meurtre sur le compte de la folie. A la faille traditionnelle du héros tragique, Shakespeare ajoute la dimension du péché et les faiblesses de l'âme.

Cette relativité apparente et artificielle du mal se retrouve dans les transgressions faites aux lois des hommes. Ce qui implique la notion de justice, cette justice des hommes si facile à acheter comme le rappelle le roi (III.3.57-60). Les hommes, les princes peuvent donc s'approprier le droit de dire ce qui est bon et ce qui est mal. La valeur s'établit en fonction de critères instables au gré des idéologies et tous les excès sont justifiables, excusables. Claudius condamne Hamlet au nom de la raison d'État, Guildenstern et Rosencrantz abondent dans son sens, soutenant que l'intérêt général prévaut sur l'intérêt particulier et sur les liens familiaux. Le crime alors devient licite. Le vieil Hamlet provoquant en un duel épique le vieux Norvège tue son adversaire et l'assassin se métamorphose en héros. La frontière qui sépare le héros de l'assassin devient incertaine, aléatoire. Presque tous les personnages sont tour à tour espions et espionnés, c'est alors l'univers des stratagèmes et des intrigues qui atteint même le burlesque quand, par exemple, Polonius faisant suivre son fils explique à Reynaldo comment plaider le faux pour savoir le vrai (II.1.62). Tout ceci n'est finalement qu'accessoire, car le thème principal est bien la vengeance. Et rendre le mal pour le mal, pose nécessairement la question de savoir d'où vient le mal, c'est-à-dire de savoir qui a commis la faute initiale. La pièce n'apporte pas de réponse, au contraire elle met en branle un système de rouages qui ne s'arrête qu'avec la mort des acteurs du drame.

\section{B. Philosophie du mal}

Dans cette perspective, le mal est, pour citer Lalande, «tout ce qui est objet de désapprobation et de blâme», par rapport aux lois de Dieu et aux lois des hommes. Pour donner quelque consistance à cette notion du mal dans l'absolu mis en question par Hamlet lui-même, on peut dire que c'est ce qui est contraire à la vertu, ou à la nature (I.3.38-40). Contrairement aux sages antiques qui tentaient de dissiper l'angoisse de l'homme devant le mal, Shakespeare, dans Hamlet, tend à multiplier les interrogations. La seule réponse vient de Horatio, le stoïque qui semble admettre que le mal, comme les calamités naturelles qui frappent les hommes et le monde «ne dépendent pas de nous [et] puisque, relevant des lois de la nature [elles sont] inscrites dans l'ordre du monde» ${ }^{4}$. Horatio tente de nous convaincre que la souffrance et la mort font partie de la condition humaine et doivent être acceptées comme 
faisant partie d'un tout. La tragédie du jeune Hamlet résiderait alors dans son refus d'une société organisée en dehors de lui et dans sa vision étroite d'un monde disloqué («out of joint») dont il n'a pas la maîtrise, reconnaissant d'abord la faiblesse de la nature humaine et la force des passions, en particulier la passion amoureuse («the ecstasy of love») qui, telle une maladie, affecte notre nature (II.1.105-7). Le mal détruit l'individu comme il détruit les États, et dans Hamlet le bien ne triomphe pas, même si c'est avec la bénédiction d'un Hamlet mourant que l'ennemi héréditaire, Fortinbras, rétablit l'ordre, un ordre qui ne peut être que fragile. La réflexion, la philosophie ne résolvent donc pas les problèmes, mais elles ne font que les poser. Le mal dépasse la réflexion philosophique, et comme le constate Hamlet: "There are more things in heaven and earth, Horatio, / Than are dreamt of in our philosophy» ${ }^{5}$ (I.5.174-5).

La théologie chrétienne à laquelle Shakespeare ne fait que très discrètement allusion dans Hamlet ne nous apporte pas davantage de solutions. Comme l'écrit E. Borne, on passe de la «cosmodicée stoïcienne à la théodicée chrétienne». Si on met à part le premier récit de la création dans lequel le mal n'apparaît pas explicitement, le problème du mal dans le second se pose par rapport à un Dieu responsable de sa création, donc responsable aussi du mal préexistant dont l'emblème est l'arbre de la connaissance. D'où le recours à Dieu pour se délivrer du mal (Matt.6.13). Dieu a le pouvoir de transformer le mal en bien, c'est par exemple dans Hamlet la paix retrouvée, même si elle est douteuse. Le mal serait ainsi la condition du bien, ce qui justifie le sacrifice d'Ophélie, martyre équivoque, à la fois victime et porteuse du mal. «Le mal est manque et privation, donc absence d'être d'où il suit que Dieu qui est l'Être et le créateur des êtres ne saurait être appelé à répondre de ce non-être qu'est le mal... L'enfer n'est infernal que par une absence, il est vrai, éternelle, celle de l'amour» ${ }^{6}$. L'enfer n'est pas les autres comme l'assure la philosophie existentialiste, l'enfer n'est pas soi-même comme le crie le Docteur Faust, l'enfer c'est l'absence, le vide, le néant. La métaphore de ce non-être est le fantôme, il est «la chose» («the thing») ou plus exactement il n'est «rien» («nothing»).

\section{Les conséquences de la conscience du mal}

La conscience du mal chez Claudius se traduit par un cynisme actif qui joue sur l'évaluation du bien et du mal d'un point de vue politique (IV.3.6-7 ; IV.7.20-1 \& III.1.48-55). Agent du démon, il joue jusqu'au bout son rôle de scélérat. Il maîtrise les situations et programme froidement l'exécution de son neveu. La lucidité ne conduit donc pour lui ni aux remords ni au repentir. En revanche Gertrude est accessible à la contrition, donc au pardon quand elle prend conscience de sa faute, quand elle voit les taches noires, indélébiles qui marquent son âme : 
O Hamlet, speak no more.

Thou turn'st mine eyes into my very soul,

And there I see such black and grainèd spots

As will not leave their tinct.

(III.4.80-3)

Hamlet éprouve après coup une sorte de repentir et regrette d'avoir tué Polonius, mais ici comme ailleurs il s'abrite derrière la volonté de Dieu qui a voulu faire de chacun le châtiment de l'autre :

For this same lord,

I do repent. But heaven hath pleased it so,

To punish me with this, and this with me,

That I must be their scourge and minister.

Le rôle de Hamlet, encore une fois, est équivoque dans la mesure où il est à la fois le bras de la justice et celui qui subit la sentence.

Pour Hamlet, si la vengeance, forme primitive du mal, n'est pas mise en question, sinon pour la rendre plus complète, ou plus efficace, le mal en revanche est l'objet de multiples interrogations. Et c'est de l'interrogation sur l'existence du mal que naît le mal. «Le mal, en effet, ne saurait se montrer sans être aussitôt dialectiquement mis en question, et, de plus, il n'y a du mal dans le monde et dans l'histoire que parce qu'il y a pour l'homme un problème du mal» ${ }^{7}$. Hamlet pose la question du mal de manière presque obsessionnelle. La conséquence en est la mélancolie ou maladie de l'âme qui se manifeste par le refus du monde et de l'action. La conscience, c'est-à-dire la possession du sens du bien et du mal, paralyse et fait de nous tous des couards : «Thus conscience does make cowards of us all» (III.1.84).

S'en remettre à Dieu comme l'ordonne le vieil Hamlet pour le châtiment de Gertrude est une sorte d'abdication, et rejeter la responsabilité du mal sur Dieu tel que cela est présenté dans la pièce relève de la chicane et du sophisme. Accepter le mal comme le fait Horatio est réservé aux sages (III.2.58-60). Idéal envié, mais inaccessible pour Hamlet dont le modèle est peut-être ce philosophe qui n'est pas esclave de la passion (III.2.66-7). Il reste deux portes étroites, celle de la folie, réelle ou feinte, et celle du suicide. L'une et l'autre sont un refus extrême d'aborder le problème. La folie ravalant la condition d'homme à celle des bêtes (IV.5.80-2), et le meurtre de soi-même faisant que l'homme se substitue à Dieu. Folie et suicide sont si étroitement liés dans la tragédie qu'ils arrivent à se confondre. La tentation du suicide est clairement évoquée chez Hamlet qui se soucie de sa vie comme d'une guigne, qui croit à 
l'immortalité de l'âme (I.4.43-6), et par conséquent ne prend guère de risques. Mais dans son infinie perversité, Dieu a interdit qu'on se tue soi-même «[O] that the Everlasting has not fixed / His canon 'gainst self slaughter» (I.2.12930 ) et l'homme est donc condamné à vivre dans sa chair souillée qui refuse de se dissoudre et de se fondre en rosée (I.2.131-2).

\section{ESTHÉTIQUE DU MAL}

\section{A. Prédestination et tragédie}

Si comme dans Coriolan on considère que les dieux jouent avec les hommes comme les enfants pervers déchiquettent les mouches, la responsabilité du héros tragique est quasiment nulle. Tout se joue en dehors de lui. Ce schéma qui prévaut dans les drames imités de Sénèque et qui se perpétue en partie dans les drames de la vengeance à l'époque élisabéthaine, est profondément modifié dans Hamlet. Le mal qui frappe le monde et les hommes n'est plus l'apanage de Dieu ou des dieux, mais souvent le fait de l'homme lui-même. Le paradoxe est que l'homme, comme Faust ou Tamerlan, voulant se mesurer aux dieux ou aux démons, se retrouve poussière. Hamlet fait lui aussi l'apologie de l'homme tout puissant, cette merveilleuse machine et constate en même temps son néant :

What a piece of work is a man, how noble in reason, how infinite in faculty, in form and moving how express and admirable, in action how like an angel, in apprehension how like a god - the beauty of the world, the paragon of animals! And yet, to me, what is this quintessence of dust?

$$
\text { (II.2.297-306) }
$$

L'homme est poussière, mais c'est le fait de pouvoir distinguer le bien du mal qui l'arrache à la condition de bête (appendice xiii après IV.4.8, vers 24-31, p. 363-4, texte Q2).

C'est la prise de conscience de ses capacités et de ses défauts qui fait la grandeur et la faiblesse de l'homme nouveau. Dans Hamlet, les rois et les reines les plus machiavéliques ne sont que des êtres humains, qui seuls avec eux-mêmes sont en proie aux pires tourments de l'âme, comme Claudius : «My soul is full of discord and dismay» (IV.1.40), ou Gertrude : «To my sick soul, as sin's true nature is, / Each toy seems prologue to some great amiss» (IV.5.17-19). La volonté, la maîtrise de soi peuvent changer la nature de l'homme, peuvent dompter le diable ou l'exorciser (appendice ix, III.4.159, vers $1-4$, p. 360, texte Q2). L'homme évite ainsi d'être un jouet entre les 
mains des dieux, d'être esclave de ses passions. Sa capacité à juger entre le bien et le mal fait de lui à la fois un dieu et un démon. Le but à atteindre est de mettre en parfaite adéquation les impératifs de sa conscience avec ses actes. Il y a là l'immense privilège du libre arbitre, mais aussi la redoutable et diabolique faculté de juger les autres.

\section{B. Dramaturgie du mal}

Les liens entre la scène et le mal sont particulièrement étroits dans Hamlet, non seulement sur le plan théâtral, sur le plan de la représentation, mais aussi sur le plan moral. Le théâtre en dénonçant le mal est censé faire triompher le bien, servir la vérité et permettre de révéler la conscience des justes comme de démasquer celle des méchants. Cette fonction du théâtre est soulignée par Hamlet (II.2.552) qui croit aux vertus de la scène pour faire avouer les coupables (II.2.577-81), le théâtre devenant alors le piège des consciences : «The play is the thing / Wherein I'll catch the conscience of the King (II.2.593-4)

La représentation du mal, tradition héritée des Mystères et des Diableries du moyen-âge, est une part essentielle des éléments spectaculaires dans Hamlet. Le fantôme peut être pris pour un messager du démon, ni homme ni esprit, il est la chose et la non chose (I.1.21), l'être et le non-être. Il est le dénominateur commun entre le ciel, la terre et l'enfer (I.5.92-3). Tel un démon, ou un acteur, il peut prendre des formes différentes (I.4.48 \& 51), alors le secours ne peut venir que des anges gardiens et des chérubins (III.4.967). Claudius est, aux yeux d'Hamlet, l'archétype du mauvais roi, l'incarnation du mal, «a vice of king» (III.4.90), le scélérat tel qu'on le trouve dans les Moralités et les Interludes. Mais dans Hamlet, comme dans Faust, les images du mal ne sont que de simples ornements.

Le mal est parfaitement intégré à l'action. C'en est même le ressort principal, le mal appelle le mal, c'est ainsi que le cycle de l'action est engagé. La cruauté devient un jeu à la limite de la perversion, et Hamlet va au-delà de sa mission quand, avec sa mère, il veut être cruel mais non contre nature : «Let me be cruel, not unnatural. / I will speak daggers to her, but use none» (III.2.378-9). Hamlet est un drame à multiples facettes ou plutôt à multiples enchâssements, car les interférences sont nombreuses. Si on veut y voir une simple allégorie de la vertu triomphant du mal, c'est évidemment oublier la dimension ironique et ambiguë du dénouement. Shakespeare utilise de façon complexe le principe simple de l'arroseur arrosé, si courant dans les fabliaux : que ce soit Guildenstern et Rosencrantz pris à leur propre piège, Laertes et Claudius empoisonneurs empoisonnés, ou la cohorte des assassins assassinés.

Pour éclairer ce sinistre tableau, Shakespeare ajoute une dimension burlesque du mal définitif qui est la mort et les morts. La scène du cimetière en est une illustration. Un des fossoyeurs fait une confusion révélatrice entre 
rédemption et damnation (V.1.1). Le mal est donc dans une certaine mesure démystifié et démythifié, et les repères déjà flous sont encore plus brouillés.

\section{La beauté du mal}

Tout interdit appelle la transgression, et toute transgression semble avoir du charme. Le serpent, agent du mal et le mal lui-même, réussit à séduire Ève. Claudius est le serpent mythique source du mal, comme le rappelle le vieil Hamlet à son fils : «A serpent stung me... The serpent that did sting thy father's life / Now wears his crown» (I.5.35-39). Il est aussi le serpent qui a séduit Gertrude. La représentation des damnés sur les tympans des cathédrales est aussi esthétique et peut-être plus saisissante que la représentation des justes. L'adage veut qu'on ne fasse pas de bonne littérature avec de beaux sentiments. Les tragédies sont peuplées de méchants, Ophélie, Cordélia ou Malcolm servent de repoussoir au mal qui les submerge. Il n'est donc pas surprenant que le dramaturge souligne les effets dévastateurs de la passion sur des hommes et des femmes qui en sont les esclaves. Sous couvert de le dénoncer, le mal est décrit avec une certaine complaisance qui implique l'irrésistible séduction du péché, qu'il soit véniel ou capital. Le mal a sa beauté comme Baudelaire le démontre dans son projet de préface aux Fleurs du mal, intitulé justement «La beauté du mal».

A la mythologie conventionnelle du mal présente dans Hamlet, s'ajoutent de nouvelles paraboles. Le mal est en quelque sorte mis en abyme, en particulier la représentation de la violence dans sa forme la plus absolue, celle des meurtres et de la mort. Il s'agit surtout d'images, car la référence est d'abord théâtrale: «This play is the image of a murder done in Vienna» (III.2.223-4). Il y a un système d'enchâssements, chaque allusion ou chaque insert reprenant, multipliant les effets, ainsi en est-il du meurtre de Priam par Pyrrhus (II.2.437-9), du meurtre de Gonzague par Lucianus déjà représenté une première fois par la pantomime. Cette technique de mise en valeur du mal s'apparente aux effets spéculaires si évidents dans la pièce, que ce soit des effets stylistiques comme l'image de l'offense renvoyée au coupable :

Ger. Hamlet, thou hast thy father much offended.

Ham. Mother, you have my father much offended.

(III.4.10-1),

que ce soit le miroir tendu par Hamlet à sa mère, ou que ce soit le théâtre luimême qui sert à révéler le mal.

Le mal prolifère dans un milieu propice, dans l'atmosphère qui règne à Elseneur et au-delà. Dans la pénombre et dans la nuit le mal accomplit sa tâche, inspire et nourrit les images de la mort. Tel un chancre insatiable, il touche et contamine le corps des hommes aussi bien que le corps de l'État. 
On ne peut nier le côté spectaculaire et même racoleur du mal dans Hamlet. Mais il y a aussi quelque chose de pathétique dans les affrontements entre Hamlet et sa mère, entre Hamlet et lui-même qui, investi de la mission de vengeur, se voit condamner à entrer dans le monde du mal. Comme son père, le jeune Hamlet semble lui aussi s'être échappé des enfers, et à travers les yeux d'Ophélie (II.1.83-5), le spectateur perçoit les ravages du mal dans la folie, autre forme du mal. Que dire de la folie d'Ophélie ? Trop faible, elle s'est laissée détruire par le mal, mais seule elle est capable de métamorphoser l'horreur en beauté :

Thought and affliction, passion, hell itself

She turns to favour and to prettiness

(IV.5.188-9)

\section{Conclusion}

Hamlet, comme ses amis et comme le spectateur, éprouve une angoisse irrépressible et tenace devant l'image du mal représentée par le fantôme. Si le mythe biblique reste équivoque sur l'origine du mal et n'en explique pas la nature, il faut en créer un autre. «Il n'y a mythe que si l'événement fondateur n'a pas de place dans l'histoire, mais dans un temps avant l'histoire... c'est le rapport de notre temps avec ce temps qui constitue le mythe». Si l'on en croit cette définition du mythe par Paul Ricœur, le fantôme, revenu de ces pays inconnus, établit ce rapport dans l'espace et dans le temps. De nouvelles images de mort, de cadavres dévorés par les vers qui font penser aux visions de Jérôme Bosch se substituent aux images d'un paradis perdu. Leurs qualités esthétiques et leur impact dramatique en sont d'autant plus forts. Le mal fait partie intégrante de la vie quotidienne et de l'existence. Hamlet se rend compte que le mal a existé avant sa propre histoire, que d'autres rois se sont fait la guerre, que d'autres frères ont tué leur propre frère. Mais le mal est si proche que lui, qui est à la fois le fils, le prince, le neveu et l'ami, Hamlet doit en prendre sa part.

Face au mal, Hamlet est-il victime ou maître de son destin ? Le texte ne nous donne pas de réponse claire. Comme dans la tragédie antique, les dieux lui imposent une mission impossible pour lui. Le destin, dans cette perspective l'exonère, l'absout du mal qu'il peut faire, mais est-ce le hasard seul qui est la cause de la mort de Polonius? «Une vraie question peut être résolue, un faux problème doit être dissout» ${ }^{8}$. Il semble que Shakespeare penche pour la deuxième hypothèse et laisse aux vers (ceux du poète autant que ceux de la terre !) le soin d'effacer définitivement les traces du mal.

Hamlet n'est pas un traité philosophique ou moral, c'est avant tout une pièce de théâtre et c'est sur le plan esthétique et dramatique qu'elle mérite d'être appréciée, même si, ou peut-être justement parce que, le mal est le lieu 
de convergence de toutes les intrigues. «Poussant le mal à l'absolu d'une manière exactement spectaculaire, le tragique poétiquement représenté en propose aussi une sorte d'absolution par recours au destin qui enveloppe les dieux et les héros, les crimes et les malheurs, dans une totalité nécessaire et belle au sein de laquelle les antagonismes se compensent et s'annulent de façon qu'au terme l'injustifiable apparaisse - mais ce n'est qu'une apparence - comme absolument justifié» ${ }^{9}$. On a dit que le mal est un défi à la raison, dans Hamlet la raison lance un défi au mal. Claudius cherche même par quelques subtiles arguties à obtenir le pardon de l'impardonnable et Hamlet tente de rendre la folie responsable du mal, mais ce sont finalement les interrogations et les doutes qui sont les plus convaincants. Hamlet échoue dans sa double exigence de faire coïncider l'être et la vertu ${ }^{10}$, cet échec éclate avec l'hécatombe finale, c'est le triomphe du mal. Il n'y a pas éradication, et l'histoire ne fait que recommencer.

Jean-Pierre VILLQUIN Université de Nantes

\section{${ }^{\mathrm{N}}$ O T E S}

${ }^{1}$ Le texte utilisé est celui de la collection World's Classics, G. R. Hibbards ed., Oxford University Press, Oxford, 1987.

${ }^{2}$ E. Borne, entrée : «mal», Encyclopaedia Universalis, Paris, 1966, vol. 10, p. 345.

${ }^{3}$ Les deux versions du «Notre Père» en français soulignent la différence : «ne nous laissez pas succomber à la tentation», et «ne nous soumets pas à la tentation».

${ }^{4}$ E. Borne, «la sagesse antique dans les formes classiques du discours rationaliste», op. cit., p. 346.

${ }^{5}$ Dans le Quarto 1 et le Quarto 2, on trouve «your» au lieu de «our» dans le Folio, ce qui ne change rien sur le fond.

${ }^{6}$ E. Borne, op. cit. p. 347.

${ }^{7}$ E. Borne, op . cit. p. 347.

${ }^{8}$ E. Borne, op. cit., p. 346.

${ }^{9}$ E. Borne, op . cit., p. 346. 
${ }^{10}$ «Cette exigence qu'atteste le suicide nihiliste lui-même qui peut vouloir que le non-être soit parce qu'il lui reconnaît valeur par rapport au mal de l'être», E. Borne, op. cit., p. 346. C'est bien là le problème que pose Hamlet. 